\title{
Common psychiatric disorders in glaucoma patients as seen at the University of Benin Teaching Hospital, Benin City, Nigeria
}

\author{
OA Dawodu $u^{a}$, AN Otakpor ${ }^{b}$ and Ukponmwan $C U^{a}$
}

\begin{abstract}
Fifty consenting patients attending glaucoma clinic were recruited over a six-month period and screened for general psychiatric morbidity, anxiety and depression using the SRQ-20 and HAD scales respectively. The male:female ratio of the group was 2.1:1 with a mean age of 57.3 years. The elderly population constituted $52 \%$ of the group. Twenty two per cent of the patients had a probable co-morbid psychiatric disorder with the prevalence rates of $10 \%$ and $6 \%$ respectively for anxiety and depression. All the depressed patients were males. The glaucoma patients with co-morbid psychiatric disorder had significantly higher scores on all psychometric test instruments than those without comorbidity. Lack of formal education was the only socio-demographic variable that was significantly associated with psychiatric co-morbidity. Patients aged 50 years or above scored significantly higher than those aged less than 50 years on the depression subscale of the HADS, while the reverse was true for the anxiety sub-scale. The implications of these findings in the management of glaucoma patients are discussed. The prevalence and pattern of distribution of psychiatric disorders found among glaucoma patients in this study did not differ from that of the normal population.
\end{abstract}

\section{INTRODUCTION}

Glaucoma is a heterogeneous group of conditions involving cupping and atrophy of the optic nerve, characteristic visual field loss and often but not invariably a high intraocular

KEY WORDS: Glaucoma patients, common psychiatric disorders, anxiety, depression

Departments of ${ }^{a}$ Ophthalmology and ${ }^{b}$ Mental Health, University of Benin/University of Benin Teaching Hospital, PMB 1111, Benin City.

Correspondence: OA Dawodu, Department of Ophthalmology, University of Benin/University of Benin Teaching Hospital, PMB 1111, Benin City. Tel: 23452 450275, 234802 3272392; E-mail: oseluesed@ yahoo.com pressure. ${ }^{1}$ Different populations tend to suffer from different types of glaucoma. The chronic simple glaucoma is the more common form of the disease in our environment (and generally in people of African descent), while the closed angle form of the disease is more common in people of Asian descent. ${ }^{1,2}$

Glaucoma is the third largest cause of blindness worldwide after cataract and trachoma. WHO estimates that about 105 million people suffer from glaucoma around the world, and an estimated 5.2 million are blind from it. ${ }^{1}$ It has been found that $24 \%$ of sufferers of chronic simple glaucoma were blind in at least one eye. ${ }^{3}$ This is because there is no reliable and cost-effective way of 
detecting and treating the disease in large populations. The burden of blindness from the different types of glaucoma is high. Therefore, making a diagnosis of a serious eye disease such as glaucoma, which can lead to blindness, usually has an emotional impact on the patient. Just as psychosocial stress can translate into illnesses that warrant treatment, ${ }^{4}$ so do some physical illnesses also create psychological sequelae that precipitate psychiatric disorders severe enough to require specialist treatment. However, a large majority of patients with physical disorder including glaucoma suffer from hidden psychiatric disorders that are often undetected by their attending primary care physicians. ${ }^{5}$ Depression and anxiety constitute greater percentage of these common co-morbid psychiatric disorders in physically ill patients. ${ }^{5,6}$ No previous research report in this locale has addressed this problem among glaucoma patients.

The aim of this study was to evaluate glaucoma patients to ascertain the prevalence of psychiatric disorders, particularly anxiety and depression. Secondly, to delineate the socio-demographic characteristics of glaucoma patients with co-morbid psychiatric disorders.

\section{MATERIALS AND METHODS}

\section{Study population}

Subjects for this study were recruited from among patients attending the glaucoma clinic at the Consultant Outpatient Department of the University of Benin Teaching Hospital (UBTH) between July and December 2002. Only those patients who gave verbal consent to participate in the study and were diagnosed as suffering from uncomplicated open angle glaucoma by the specialist ophthalmologists were included. All glaucoma patients with coexisting ocular pathology such as media opacities, retina and macular diseases were excluded.

\section{Measures}

Two main questionnaires were used for this study as measures of general psychiatric morbidity, and anxiety and depression. The 20-item self-reporting questionnaire (SRQ-20) is a World Health Organization (WHO) psychometric instrument for measuring the level of psychiatric morbidity in primary health care settings. Its norms for use among Nigerian patient populations are wellestablished, with a cut-off score of five or more discriminating between cases and noncases of psychiatric disorders among Nigerian patients. The instrument has a sensitivity of $95.8 \%$, a specificity of $90.9 \%$ and a minimum misclassification rate of $7.9 \% .^{7}$

The hospital anxiety and depression scale (HADS) developed by Zigmond and Snaith in 1983 is a 14-item instrument especially designed for use among non-psychiatric patients. It consists of seven items each for anxiety and depression, rated on a four-point scale of severity. The instrument is devoid of somatic symptoms, hence its usefulness in assessing the impact of physical illness on psychological well being. Its utility among widely varied Nigerian patients' populations and the established norms informed the choice of this instrument. ${ }^{8}$

\section{Design}

A two-stage prospective case identification technique was adopted for the study.

\section{Procedure}

All new referrals to the glaucoma clinic during the study period were evaluated by the ophthalmologist authors. Patients that met the inclusion criteria for the study were then asked to see the trained research assistant in the adjoining room. Each patient was first assessed using the SRQ-20. Those with a score of five or more were subsequently interviewed using the HADS instrument. On the average, it took 25 minutes to administer both que- 
stionnaires. The demographic characteristics consisting of age, sex, marital status, level of education and occupation were obtained from the patients using a separate data collection sheet. Simple descriptive statistics was adopted for data analysis using the Student's t and the chi-square tests where appropriate. Significant probability level was set at p < 0.05 .

\section{RESULTS}

Fifty subjects comprising 34 males and 16 females with a male:female ratio of 2.1:1 and an age range of 16 to 92 years (mean $57.3 \pm$
39.2 years) were recruited for the study. Table 1 shows the age and sex distribution of the group. It is noteworthy that the vast majority (76\%) were aged 50 years or above and that the elderly population aged 60 years or above constituted $52 \%$ of the study group. Other socio-demographic features of the study group are as shown in Tables 2 and 3.

Eleven (22\%) patients in the study group with a mean age of 49.09 years and comprising seven males and four females scored $\geq 5$ on the SRQ-20, thus giving a probable psychiatric morbidity prevalence rate of $22 \%$. The hospital anxiety and

\section{Table $1 \quad$ Age and sex distribution}

\begin{tabular}{lrrrr}
\hline Age group (years) & Male & Female & Total & \% \\
\hline$<19$ & 1 & 1 & 2 & 4 \\
$20-29$ & 3 & 2 & 5 & 10 \\
$30-39$ & 1 & 1 & 2 & 4 \\
$40-49$ & 2 & 1 & 3 & 6 \\
$50-59$ & 7 & 5 & 12 & 24 \\
$>60$ & 20 & 6 & 26 & 52 \\
\hline Total & 34 & 16 & 50 & 100 \\
\hline
\end{tabular}

Table 2 Marital status and educational attainment of the patients

\begin{tabular}{lrlr}
\hline Marital status & Number (\%) & Educational status & Number (\%) \\
\hline Married & $38(76)$ & No formal education & $9(18)$ \\
Single & $7(14)$ & Primary education & $21(42)$ \\
Separated/widow & $5(10)$ & Secondary education & $14(28)$ \\
Total & $50(100)$ & Tertiary education & $6(12)$ \\
& & Total & $50(100)$ \\
\hline
\end{tabular}

\section{Table 3 Occupational status of patients}

\begin{tabular}{lr}
\hline Occupational status & Number (\%) \\
\hline Professionals & $4(8)$ \\
Skilled/semi-skilled & $14(28)$ \\
Unskilled & $5(10)$ \\
Unemployed & $6(12)$ \\
Miscellaneous (housewife, student, retiree) & $19(38)$ \\
Unspecified & $2(4)$ \\
Total & $50(100)$ \\
\hline
\end{tabular}


Table 4 Socio-demographic characteristics and test scores of each of the eleven identified cases

\begin{tabular}{|c|c|c|c|c|c|c|c|c|}
\hline S/No. & $\begin{array}{c}\text { Age } \\
\text { (years) }\end{array}$ & Sex & $\begin{array}{c}\text { Marital } \\
\text { status }\end{array}$ & $\begin{array}{c}\text { Educational } \\
\text { level }\end{array}$ & $\begin{array}{c}\text { Occupation } \\
\text { score }\end{array}$ & SRQ-20 & $\begin{array}{r}\text { HADS } \\
\text { (anxiety) }\end{array}$ & $\begin{array}{c}\text { HADS } \\
\text { (depression) }\end{array}$ \\
\hline$\overline{1}$ & 70 & $\bar{M}$ & Married & Primary & Retiree & 5 & 6 & 8 \\
\hline 2 & 58 & $\mathrm{~F}$ & Married & Nil & Trader & 5 & 0 & 4 \\
\hline 3 & 50 & M & Married & Nil & Farmer & 9 & 5 & 10 \\
\hline 4 & 70 & $\mathrm{~F}$ & Widow & Nil & Nil & 9 & 3 & 4 \\
\hline 5 & 68 & $\mathrm{~F}$ & Widow & $\mathrm{Nil}$ & Nil & 10 & 9 & 6 \\
\hline 6 & 28 & $\mathrm{~F}$ & Single & Tertiary & Student & 9 & 10 & 4 \\
\hline 7 & 26 & M & Single & Secondary & Unemployed & 6 & 9 & 5 \\
\hline 8 & 18 & M & Single & Secondary & Student & 6 & 11 & 6 \\
\hline 9 & 56 & M & Married & Nil & Farmer & 6 & 6 & 9 \\
\hline 10 & 37 & M & Married & Secondary & Technician & 6 & 10 & 1 \\
\hline 11 & 59 & M & Married & Primary & Carpenter & 14 & 4 & 12 \\
\hline
\end{tabular}

depression scale identified five of the cases as suffering from probable anxiety disorder, three as probable cases of depressive disorder, while the remaining three failed to meet the cut-off score for either diagnostic category. Thus, the study group had probable prevalence rates of $10 \%$ and $6 \%$ respectively for anxiety and depressive disorders.

Table 4 summarises the socio-demographic characteristics and psychometric test scores of the eleven cases identified by the SRQ-20. Lack of formal education was the only factor identified to be associated with the presence of psychiatric morbidity (SRQ$20 \geq 5$ ) and significantly distinguished the cases from non-cases (Fisher's exact $\chi^{2}=$ 20.891, df $=3, p<0.0075)$. The eleven cases scored significantly higher than the non-cases in all psychometric measures. Although the four female cases identified had a mean age of $56.0 \pm 19.4$ years as against $45.1 \pm 18.8$ years for the seven male cases, the difference was not statistically significant $(\mathrm{t}=0.8026$, $\mathrm{df}=9, \mathrm{p}>0.05)$. Seven of the eleven identified cases were aged 50 years and above as against four aged less than 50 years. The cases aged 50 years and above were significantly more depressed than those below 50 years (mean HADS depression score of $7.5 \pm 3.05$ versus $4.0 \pm 2.16 ; \mathrm{t}=2.0284$, df $=9, \mathrm{p}<0.05)$. The reverse was the case with their performance on the anxiety axis of the test instrument $(\geq 50$ years versus $<50$ years test scores were $5.17 \pm 2.97$ versus $10.0+$ $0.5, \mathrm{t}=3.22, \mathrm{p}<0.01)$.

\section{DISCUSSION}

Long-term treatment of chronic illnesses could be stressful for patients. For patients with open angle glaucoma, apart from frequent and multiple hospital visits, cost of drugs, transportation and other expenses connected with management, there is also the fear of losing vision, which could be a source of anxiety or depression.

Twenty two per cent of the study population tested positive for psychiatric disorders using the SRQ-20. Since up to $25 \%$ of the normal population could test positive with this mode of assessment, it falls within normal limits. Five out of eight patients that were positive for psychiatric disorders using the HADS had anxiety, while three had depression. This also follows the pattern of distribution of these disorders when normal populations are assessed with these tests. 
Literature on the psychiatric co-morbidity in glaucoma patients is scanty. In a reported case-control study by means of self-report questionnaire, Roy Wilson et $\mathrm{al}^{9}$ found no significant difference in depression scores of glaucoma patients and controls. Noteworthy is the fact that they had more male than female representation among their glaucoma study group with the majority of them not working, and being significantly older than their control groups. Furthermore, they found $7.4 \%$ prevalence of depression among glaucoma patients as against $13.3-21.4 \%$ among their control group who had only cataract. This study and ours suffer similar methodological flaw in that questionnaires never substituted for clinical interview; yet the findings regarding prevalence of depression are strikingly similar: we found $6 \%$ prevalence rate for depression. Contrary to the findings of Roy Wilson et al, ${ }^{9}$ referred to above, Erb et $\mathrm{al}^{10}$ in their study of psychiatric manifestations in patients with primary open angle glaucoma, noted that while in-patient glaucoma patients had higher scores on depression than their outpatient counterparts and the cataract control group, the outpatient glaucoma group had statistically significant higher score for psychosomatic complaints. The score on all three parameters (depression, psycho-somatic complaints, and emotional stability) were normal for the cataract patients. Only the inpatient glaucoma group was subjected to a clinical psychiatric interview in this study, thus making comparison of prevalence rates difficult.

The use of two conceptually contrasting questionnaires in our study is noteworthy. The criticism of the SRQ as having high somatic components, which would seem to make for high sensitivity in physically ill patients, is balanced out by the use of the HAD scale, which is devoid of somatic complaints or symptoms. The instrument notwithstanding, prevalence of psychiatric disorder is known to vary markedly and this is partly dependent on the study population. In a particular primary health care centre in Nigeria, using the 12-item general health questionnaire (GHQ-12) and the SRQ-20, prevalence of psychiatric disorder was found to vary from $6.4 \%$ to $13.6 \%$ in the same patients. ${ }^{11}$ Our finding of $22 \%$ prevalence rate is similar to the $22.4 \%$ reported by Abiodun ${ }^{7}$ among his study group. That $52 \%$ of our glaucoma patients are elderly (age $>60$ years) is in keeping with the known fact about glaucoma as an illness of the aged. While depression is known to be commoner among females, we found all three depressed patients to be male in this study. The smallness of the numbers, coupled with the other finding that the psychopathologic females were older than the males, may make this a spurious finding. It is, however, to be noted for future studies. Of the psychopathologic cases identified in this study, those aged 50 years and above were more depressed while the younger people aged below 50 years suffered more from anxiety disorder. This trend of varying psychopathologic finding with age could well be the result of perceived helplessness and hopelessness among the older population suffering from an "incurable" visual problem; the initial anxiety being the result of efforts to find a final cure to a threatening visual loss after previous disappointing remedies. There is therefore a need to explore the emotional state of glaucoma patients in order to help improve the totality of their quality of life.

This study did not take into consideration the influence of duration of illness in these patients nor the extent of visual field loss prior to the study even though no statistically significant association between visual field loss and depression was found in a previous study. ${ }^{9}$ 


\section{References}

1. World Health Organization. Press Office. Fact Sheet 1997; 143: 2.

2. Johnson GJ. The adult glaucomas. Comm Eye Health 2001; 14(39): 33.

3. Foster PJ. Advances in understanding of primary angle-closure as a cause of primary glaucomatous optic neuropathy. Comm Eye Health 2001; 14(39): 37.

4. Oheari JU. Psychosocial stress and emotional disorders. W Afr J Med 1992; 11(3): 234-235.

5. Bridges $K$ and Goldberg $D$. Somatic presentation of depressive illness primary care. In: Freeling P, Downey LJ and Mackin JC (Eds.). The Presentation of Depression: Current Approaches. UK: Royal College of General Practitioners, 1987; 36, 9-11.

6. Burton RH and Freeling P. Unrecognised depression in general practice. In: Freeling P, Downey LJ and Malkin JC (Eds.). The Presentation of Depression: Current Approaches. UK: Royal College of General Practitioners, 1987; 36, 12-16.
7. Abiodun OA. Sensitivity and validity of the SRQ-20 in a primary health care centre in a rural community in Nigeria. Psychopathol Afr 1988; XX11(1): 79-88.

8. Abiodun OA. A validity study of the hospital anxiety and depression scale in general hospital units and a community sample in Nigeria. Br J Psychiatry 1994; 165(5): 665672.

9. Roy Wilson M, Coleman AL, Fei Yu, Sasaki IF, Bing EC and Kim MH. Depression in patients with glaucoma as measured by selfreport surveys. Ophthalmology 2002; 109: 1018-1022.

10. Erb C, Batra A, Bromer A, Bayer AU, Muller -Schaaf and Thiel HJ. Psychiatric Manifestations in patients with primary open angle glaucoma. Ophthalmology 1993; 90(6): 635-639.

11. Oheari JU, Odejide OA, Gureje $\mathrm{O}$ and Olatawura MO. The prevalence of psychiatric morbidity among adult attenders at the five primary health care facilities of a rural community in Nigeria. Psychopathol Afr 1994; XXVI(1): 97-108. 